\title{
Mujeres de clase popular y actividad física: limitaciones, recursos territoriales y agencia para el cuidado de sí.
}

\section{Working-class women and physical activity: constraints, territorial resources and agency for self-care.}

\author{
Betina Freidin \\ Mercedes Krause ${ }^{* *}$ \\ Matías Salvador Ballesteros ${ }^{* *}$ \\ Agustín Wilner
}

\begin{abstract}
Resumen
Analizamos la práctica de actividad física en el tiempo libre de mujeres adultas de un barrio de clase popular del Conurbano de Buenos Aires, Argentina, a partir de un estudio cualitativo con grupos focales. Hacer ejercicio regularmente les proporciona bienestar psíquico y emocional. Mejorar condiciones de salud y controlar el peso también motiva la práctica. Socializar es otro incentivo. Buscan ejercer agencia temporal para el cuidado de sí ante las demandas de reproducción del grupo doméstico y del trabajo remunerado. Si bien los espacios públicos para realizar actividad física y los predios con subvención estatal facilitan la práctica regular, enfrentan restricciones territoriales para acceder a ellos. EI abandono estatal de algunas áreas obstaculiza el desplazamiento y desalienta el ejercicio en espacios públicos. Las experiencias de inseguridad y violencia interpersonal también imponen barreras para la circulación, especialmente para las más jóvenes, evidenciando las restricciones de género para apropiarse del espacio público.
\end{abstract}

Palabras clave: Actividad física, Mujeres, Tiempo libre, Clase popular, Argentina

\footnotetext{
* Ph.D en Sociología. Investigadora del Consejo Nacional de Investigaciones Científicas y Técnicas con sede en el Instituto de Investigaciones Gino Germani y Profesora regular de la Carrera de Sociología, Universidad de Buenos Aires.

** Dra. Ciencias Sociales, Mg. Investigación Social, Socióloga, docente-investigadora de la Facultad de Ciencias Sociales, Universidad de Buenos Aires

*** Sociólogo y Doctor en Ciencias Sociales por la Universidad de Buenos Aires (UBA). Investigador del Consejo Nacional de Investigaciones Científicas y Técnicas con sede en el Instituto de Investigaciones Gino Germani (UBA) y docente de la Carrera de Sociología (UBA)

${ }^{* * * *}$ Licenciado en Sociología. Becario Doctoral del Consejo Nacional de Investigaciones Científicas y Técnicas con sede en el Instituto de Investigaciones Gino Germani de la Universidad de Buenos Aires.
} 


\begin{abstract}
We analyze physical activity in leisure time among adult women who live in a working-class neighborhood in the periphery of Metropolitan Buenos Aires, Argentina, drawing on a qualitative study we conducted with focus groups. They achieve emotional and mental wellbeing through exercising regularly. To improve health conditions and control body weight are also motivations, as well as socializing. They seek to exert temporal agency for self-care when facing the demands imposed by domestic reproduction and paid work. Although public spaces for physical activity and state-subsidized facilities facilitate regular practice, there are territorial restrictions on access to them. State abandonment of some areas hinders and discourages women to exercise in public spaces. The experiences of insecurity and interpersonal violence also impose barriers, especially for the youngest women, showing gender-based restrictions on the appropriation of public space.
\end{abstract}

Keywords: Physical Activity, Women, Leisure Time, Working Class, Argentina

Fecha de recepción: Febrero 2021

Fecha de aprobación: Junio 2021

\title{
Introducción
}

Los modos de vida estructurados por las relaciones de clase, género, raciales y étnicas, y los aspectos territoriales condicionan las posibilidades de desarrollar estilos de vida saludables en las ciudades. Entre las prácticas protectoras de la salud psicofísica se encuentra el ejercicio físico regular (World Health Organization, 2018). Si bien el aumento del sedentarismo en las ciudades se asocia con los medios de transporte, el uso de tecnología, el diseño urbano y los valores culturales, las cifras reflejan patrones de desigualdad social al interior de los países (Moore, Diez, Evenson, McGinn, \& Brines, 2008; World Health Organization, 2018). Entre los grupos más afectados se encuentran las mujeres y los sectores con menores recursos socioeconómicos. Estudios internacionales muestran que las mujeres realizan menos actividad física recreativa y deporte, y lo hacen con menor regularidad. Quienes menos ejercitan son las mujeres de clase trabajadora y que tienen hijos pequeños (Hargreaves, 1993; Hormiga Sánchez, 2015), las pertenecientes a minorías étnicas y raciales, y las adultas mayores (Abichahine \& Veenstra, 2017; Ainsworth, 2000; Brady \& Nies, 1999).

En Argentina, según los datos de la Encuesta Nacional de Factores de Riesgo del año 2013, el 54\% de la población adulta urbana es sedentaria. Linetzky, De Maio, Ferrante, Konfino, \& Boissonnet, (2013) muestran que, para el período 2005-2009, la baja actividad física se incrementó para todos los grupos poblacionales, pero el incremento fue mayor para las mujeres de menor estatus socioeconómico. Con la misma fuente, Ballesteros y Freidin (2019) documentan que las personas de menor nivel educativo, de más edad y las mujeres son quienes realizaron en mayor proporción un nivel de actividad física bajo 
durante la semana previa al relevamiento, y que, considerando el último mes, cuando realizan actividad física estos grupos lo hacen mayormente por sus responsabilidades cotidianas (domésticas, laborales y de traslado). Analizando la encuesta sobre Estratificación y movilidad social del año 2007, Gómez Rojas y Riveiro (2015) observan que mientras el $25,7 \%$ de la clase de servicios nunca realiza actividad física en su tiempo libre, en la clase obrera este porcentaje asciende a $49,3 \%$, y en este último grupo las desigualdades de género son muy marcadas, ya que el $57,4 \%$ de las mujeres no realizan ejercicio frente al 40,4\% de los hombres. Existe también una alta disparidad territorial en la realización de actividad física recreativa: en el año 2016, el 81\% de los y las residentes en áreas urbanas más precarias no la habían realizado en la última semana, frente al $52 \%$ de los y las que disponían de mejores condiciones de residencia (ODSA, 2017). Sintetizando, los datos agregados muestran patrones persistentes de inequidad social en la realización de actividad física en el tiempo libre.

Por su parte, estudios cualitativos internacionales con mujeres de clase popular y minorías étnicas y raciales dan cuenta de la percepción y los significados del ejercicio físico y el tiempo libre en el contexto de sus actividades cotidianas y responsabilidades domésticas. Según el estudio de Tortolero, Mâsse y Fulton (1999) con mujeres hispanas y afroamericanas adultas en EEUU, ellas identifican las actividades domésticas y de cuidado como ejemplos de actividad física que realizan cotidianamente, y perciben el deporte como una actividad orientada a los hombres, a sus hijos y a la actividad profesional. Pocas realizan ejercicio físico recreativo de manera regular; contar con tiempo "libre" suele considerarse un lujo que está fuera de su alcance y experimentan con cierta culpa tomarse un tiempo para ellas mismas (Tortolero et al., 1999, p. 138-139). Practicar un deporte puede parecerle a las mujeres pobres algo periférico frente a sus preocupaciones cotidianas por la supervivencia material; así es que cuando, mediante iniciativas comunitarias y programas locales se abren oportunidades para el acceso al ejercicio, estas intervenciones para la promoción de la salud resultan en un empoderamiento de las mujeres más postergadas socialmente (Burnett, 2002; Hargreaves, 1993).

Son escasas las investigaciones con mujeres sobre actividad física y salud en Argentina y, como sucede con los antecedentes internacionales, se trata de trabajos cuantitativos (Hormiga Sánchez, 2015). Las investigaciones cualitativas se han centrado en mujeres y varones de clase media (Ballesteros et al., 2016; Lehner \& Ponce, 2018). En este artículo nuestro objetivo es analizar y comprender el lugar que ocupa el ejercicio físico recreativo y para el cuidado de la salud en la vida cotidiana de un grupo de mujeres adultas residentes en un barrio de clase popular del Conurbano norte de Buenos Aires, Argentina. Consideramos aspectos de la organización del hogar y del trabajo extradoméstico, económicos y barriales que facilitan o bien limitan la práctica regular de actividad física en el tiempo libre. Los datos provienen de un estudio cualitativo que realizamos con grupos focales durante los años 2015 y 2016. 


\section{Mujeres y actividad física: relaciones de género, clase social, territorio y cultura}

Los estudios sobre género y salud enfatizan una perspectiva interseccional para explicar cómo la clase social, junto con otros determinantes sociales, estructura la exposición y vulnerabilidad de hombres y mujeres a la enfermedad y el acceso a recursos protectores de la salud (lyer et al., 2008). La persistencia de las disparidades de género en la realización de ejercicio físico en el tiempo libre se explica por la subordinación de las mujeres en el manejo de recursos económicos, la menor capacidad de disponer de tiempo libre debido al doble rol económico y reproductivo, y la existencia de una organización sexista del deporte que destina mayor infraestructura y recursos a la práctica deportiva de los hombres; además, por la violencia simbólica que se ejerce sobre las mujeres, al estar muchos deportes asociados a los valores de la masculinidad hegemónica como la competencia, el éxito, la fuerza y la resistencia física (García Ferrando \& Llopis Goig, 2017; Hargreaves, 1993; Martín et al., 2017; Mosquera González, 2014). Por otra parte, la misma idea de ocio se encuentra masculinizada, es decir, definida por oposición al trabajo remunerado, siendo para las mujeres difícil distinguir su trabajo no remunerado del tiempo de ocio familiar (Hargreaves, 1993). Las madres solteras o separadas sufren desventajas para disponer de tiempo libre ya que la responsabilidad económica y de cuidado de los niños recae mayormente sobre ellas (Hargreaves, 1993). Hargreaves remarca que las desigualdades de clase acentúan las de género en el ocio y el deporte, debido a que "la mayoría de las limitaciones del ocio femenino afectan más a las mujeres de la clase trabajadora que a las de clase media-éstas últimas tienen más probabilidades de disfrutar de ayudas en la casa y en la crianza de los hijos y disponen de más dinero para el ocio y el transporte privado" (1993, p. 120). Las mujeres pobres con hijos pequeños suelen caminar grandes distancias para sus necesidades de consumo y actividades diarias, por falta de automóvil propio y mayores dificultades para acceder a la red de transporte público, lo que puede generar estrés y fatiga corporal en lugar de los beneficios psicofísicos asociados con la caminata (Bostock, 2001; Jirón Martínez, 2017). En palabras de Bostock "en efecto, las madres usan sus cuerpos como un medio para superar la brecha entre sus responsabilidades y recursos". (2001, p. 16),

En Latinoamérica las mujeres tienen mayor representación entre las personas sin ingresos propios o insuficientes para una vida digna, están sobrerrepresentadas en los hogares pobres, presentan bajas tasas de participación económica y altas tasas de desempleo, y tienen una importante sobrecarga de trabajo doméstico y de cuidado no remunerado (Segovia \& Rico, 2017). No solo ganan menos que los hombres, sino que tienen más responsabilidades de cuidado en el ámbito familiar, lo que produce "un círculo vicioso entre pobreza monetaria y pobreza de tiempo propio" (Hargreaves, 1993, p. 51). Las cargas de cuidado varían a lo largo del ciclo familiar, pero también lo hacen según la composición del hogar y los ingresos, en la medida en que dependan de la capacidad de comprar los servicios de cuidado de otras mujeres en el mercado (Esquivel, 2011; Batthyány, 2010). Disponer de tiempo es un determinante social de la salud, al ser un 
recurso multidimensional que afecta las posibilidades de desarrollar hábitos de vida saludables, incluyendo el ejercicio físico (Strazdins et al., 2015). Si bien la intensificación de las actividades y la aceleración del ritmo de vida es una experiencia compartida por gran parte de la población en las sociedades capitalistas contemporáneas, son los grupos con mayor desventaja estructural quienes tienen menor control sobre cómo transcurre su tiempo $\mathrm{y}$, por lo tanto, poseen menor capacidad de agencia temporal en su vida cotidiana. Las mujeres de clase popular tienen menor control y flexibilidad en su actividad laboral, y escasa capacidad para comprar el tiempo de otros para liberar tiempo personal (Boersma, 2016).

En contextos de pobreza, el acceso a ámbitos privados para la realización de ejercicio está restringido por la disponibilidad de dinero, además de la accesibilidad geográfica y la disponibilidad de tiempo libre. El menor acceso a espacios verdes y recreativos públicos se ve afectado por la desigual distribución de infraestructura urbana y prioridades de inversión que crean desventajas territoriales para los grupos que viven en áreas marginalizadas (Diez Roux \& Mair, 2010; Freidin et al., 2018; Moore et al., 2008). Las desventajas socioterritoriales también limitan más a las mujeres que a los varones. Siguiendo a Segovia y Rico (2017), el espacio urbano no es neutro, sino que contiene y expresa relaciones de poder, puesto que la inseguridad y la violencia interpersonal conllevan connotaciones diferentes para varones y mujeres. Para ellas, la realización de actividad física en la vía pública implica agenciarse el "derecho" de acceso, participación y uso de un espacio estructurado y restringido por el género (Allen-Collinson, 2011).

De manera complementaria a los estudios sobre desigualdad social y género, desde una perspectiva sociocultural se busca comprender las motivaciones de las mujeres para realizar actividad física (Markula \& Kennedy, 2011). Esta línea analítica ha sido desarrollada desde perspectivas feministas que deconstruyen las imágenes dominantes difundidas por los medios, según las cuales el cuerpo delgado y tonificado es sinónimo de buena salud (Markula \& Kennedy, 2011; Lupton, 1997). Algunas mujeres (al igual que algunos hombres) conciben la realización de actividad física como un trabajo sobre sí mismas, "algo bueno para mí", alineando sus motivaciones con valores de la modernidad tardía asociados con la autonomía, la racionalidad y la responsabilidad individual. Se trata de una dinámica de healthcization de prácticas corporales y consumos que contribuye a crear una moralidad en la que se combinan valores culturales y comportamientos individuales con preocupaciones y definiciones biomédicas (Conrad, 1994). Si bien son los sectores medios profesionales los que mayormente adoptan la "nueva conciencia de la salud" cristalizada en estilos de vida normativamente construidos como deseables (Crawford, 2006), las recomendaciones y prácticas atraviesan las fronteras de clase (Cockerham, 2010). "Culpabilizar a la víctima" suele ser una respuesta cuando se juzga a quienes, encontrándose en circunstancias de vida muy desventajosas, no siguen dicho mandato sociocultural (Lupton, 1997). 


\section{Metodología}

El análisis se basa en un estudio cualitativo con seis grupos focales que realizamos durante los años 2015 y 2016 con mujeres residentes en un barrio de clase popular del segundo cordón del conurbano de Buenos Aires ${ }^{1}$. En los grupos abordamos varias temáticas vinculadas con el cuidado de la salud. En este artículo nos centramos en los aspectos relativos a la actividad física.

El barrio seleccionado presenta una importante heterogeneidad socioterritorial, distinguiéndose áreas según el tipo de viviendas (la mayoría con paredes y pisos de material, pero algunas de ellas son precarias, tipo casilla), y el trazado urbano (unas pocas calles no están asfaltadas y algunas pequeñas áreas no cuentan con trazado de manzanas). Además, algunos terrenos y calles son inundables. Un canal parcialmente entubado divide al barrio a lo largo diferenciando al "lado mejor" del "lado peor" según las palabras de las mujeres que participaron de los grupos, casi en su totalidad residentes del "lado peor". Esta última área tiene mayores desventajas en la accesibilidad al transporte público y vías de acceso a rutas y autopistas, y mayores distancias a espacios públicos recreativos, incluyendo plazas y el polideportivo municipal. Concentra además mayores niveles de inseguridad por violencia callejera según la experiencia de las participantes.

La investigación cualitativa permite el acceso a los aspectos contextuales, económicos, culturales, familiares y comunitarios que influyen sobre la realización de actividad física, obteniendo testimonios personales en profundidad (Hormiga-Sánchez, 2015). Los grupos focales constituyen conversaciones colectivas sobre un tema y brindan conocimiento sobre las actitudes y creencias que subyacen a los comportamientos y sobre los contextos de las prácticas, permitiendo comprender las experiencias de una manera más holística; y son especialmente indicados para dar voz a los grupos sociales postergados (Carey \& Asbury, 2012). La interacción grupal facilita alcanzar mayor amplitud en la información reunida, y el intercambio de perspectivas, una mayor comprensión de las experiencias (Carey \& Asbury, 2012). La orientación epistemológica que seguimos es interpretativa, para dar cuenta de la variabilidad de significados sobre la temática investigada, buscando analizar y comprender en profundidad aspectos cognitivosracionales, así como emociones y construcciones intersubjetivas (Freidin, 2016).

La muestra fue de tipo intencional, a partir de un recorte etario de mujeres adultas de entre 18 y 60 años residentes en el barrio. Las mujeres fueron convocadas para participar de los grupos mediante contactos entablados por el equipo de investigación con distintas organizaciones barriales. Debido a la estrategia seguida, casi todas las mujeres

\footnotetext{
${ }^{1}$ La investigación fue acreditada y financiada por la Secretaría de Ciencia y Técnica de la Universidad de Buenos Aires (Proyecto 20020130100594, "Desigualdad social, cultura y salud: recursos y prácticas cotidianas frente al imperativo del cuidado", dirigido por Betina Freidin en el Instituto de Investigaciones Gino Germani).
} 
que participaron de los grupos se conocían previamente. Los grupos estuvieron compuestos por entre cinco y diez mujeres, totalizando 39 participantes. La edad promedio fue de 33,7 años. Casi tres cuartos de ellas eran madres (29) y tenían entre 1 y 9 hijos, con un promedio de 3 hijos. Dos tercios de ellas trabajaban fuera del hogar (26), principalmente como empleadas domésticas y de limpieza en oficinas, cocineras en una cooperativa y en un comedor comunitario, y una de ellas tenía un micro-emprendimiento hogareño junto con su marido para la elaboración de comidas. Algunas también brindaban apoyo escolar a niños, coordinaban grupos en el barrio por su formación en psicología social comunitaria, y una participante era maestra estimuladora. De las mujeres que no trabajaban remuneradamente (13), la mayoría eran amas de casa, pero también algunas estaban completando estudios secundarios en un bachillerato para adultos y, en un caso, la universidad; una mujer era pensionada con certificado de discapacidad, y otra estaba buscando trabajo. Con relación al perfil educativo, un cuarto de las participantes alcanzó hasta el nivel primario completo, un poco más de la mitad contaba con estudios secundarios incompletos, cuatro habían finalizado sus estudios secundarios y tres contaban con estudios terciarios.

Para moderar los grupos utilizamos una guía de pautas semiestructurada que incluía preguntas sobre ejercicio físico, aunque el tema emergió espontáneamente al inicio de las conversaciones en varios de los grupos cuando preguntamos a las participantes qué les parecía importante hacer para cuidar la salud. De manera complementaria a la guía, utilizamos como disparador un poster de una campaña gráfica del Plan Argentina Saludable del Ministerio de Salud de la Nación, cuyo slogan era "una vida saludable está a tu alcance"2. Entre los hábitos promovidos en la gráfica se indicaba "sumar 30 minutos diarios de movimiento", especificando "caminar más cuadras, subir y bajar escaleras, salir en bicicleta, bailar y jugar con los chicos”. La moderación de los grupos estuvo a cargo de la directora de la investigación. Los encuentros tuvieron una duración aproximada de dos horas, y, con la autorización de las participantes, fueron grabados digitalmente y desgrabados verbatim.

Resguardamos el anonimato de las participantes cambiando sus nombres. Tampoco identificamos el barrio ni la localidad. Este procedimiento fue garantizado en la nota de consentimiento informado leída al inicio de cada encuentro y entregada por escrito a las participantes. Mediante un breve cuestionario aplicado al inicio, recabamos información sociodemográfica de las mujeres para contextualizar sus intervenciones durante el análisis de los datos. Seguimos la estrategia de análisis temático, con sucesivas rondas de codificación, agrupando los códigos en núcleos temáticos o categorías de mayor abstracción para dar sentido conceptual a los datos. Utilizamos el programa ATLAS.ti para la codificación, y, de manera complementaria, elaboramos matrices cualitativas para

${ }^{2}$ La campaña no tuvo difusión masiva. Utilizamos la gráfica quitándole el texto que indicaba que era parte de una campaña oficial. 
Revista Punto Género N. 15 Junio de 2021

ISSN 0719-0417 / 1- 24

sistematizar los emergentes de cada grupo y las comparaciones entre grupos a partir de los núcleos temáticos más generales (Huberman \& Miles, 1994; Freidin, 2017b)

\section{Ejercicio y bienestar emocional: agencia para el cuidado de sí, recursos y apoyo social}

Varias de las participantes realizan actividad física de manera regular en su tiempo libre porque lo consideran muy beneficioso para su bienestar psíquico y emocional. Es el caso de Carla (41 años, empleada doméstica, madre de tres hijos adolescentes) quien, tras una crisis de pareja que la llevó a tomar una sobredosis de psicofármacos que requirió su hospitalización, comenzó a caminar cotidianamente hasta una plaza que se encuentra a diez cuadras de su casa para allí ejercitarse con los aparatos. Ella sostiene: "aunque no creas, es como una terapia hacer gimnasia" (Grupo 2). Mara (31 años, casada con 2 hijos pequeños, y coordinadora de grupos), luego de ver el afiche con la recomendación de ejercicio físico diario, acuerda con el consejo y comenta que le encanta caminar porque la distrae de sus preocupaciones cotidianas, "yo vuelvo liberada cada vez que me voy a caminar a la mañana. Auriculares, caminata, vuelvo y soy otra persona. Voy hasta la plaza [de una localidad próxima] o más lejos, depende de cómo esté enroscada ese día, y vuelvo. Puedo arrancar el día de ahí (...) me encanta" (Grupo 4). Pilar (34 años, empleada doméstica, casada y madre de tres hijos pequeños) que hacía aquagym en el polideportivo municipal también expresa que "te reconforta saber que hacés algo que es para vos, para tu cuerpo (...). Más allá de que laburar y hacer cosas en tu casa... hacer algo para vos es como... yo lo hacía a la mañana y era como que parecía que el día empezaba diferente, no sé, venís como renovada" (Grupo 6). En el Grupo 5, Margarita (52 años, vive con su esposo e hijo de 12 años) también cuenta que a ella ir al polideportivo la "despeja" y que se divierte socializando con amigas. Concurre cuatro veces por semana, a pesar de la mala situación económica de su hogar debido al desempleo prolongado de su marido y la precariedad de su trabajo, que consiste en la preparación de comidas para la venta en el barrio. Hacer actividad física recreativa le permite suspender sus preocupaciones por un rato, aun cuando su jornada de trabajo sea muy extensa y desgastante físicamente:

Moderadora- Vos decías que te despeja [ir al polideportivo]. Margarita-. Y si no, no podría llevar toda esta situación, yo ahí me olvido de todo... Bailamos, hacés gimnasia y no te das cuenta, porque es una con baile, hay varias cosas, ¿no? Y ahí te olvidás, tengo mil problemas, pero ahí te olvidás, te matás de risa, te olvidás de todo, hacés de cuenta que no tenés ningún problema. Después siguen igual, pero... (...) Yo voy cuatro veces en la semana. Moderadora- $O$ sea que también tenés que tener tiempo para ir. Margarita- $Y$, no tengo tiempo, porque estoy levantada, por ejemplo, desde las seis y media de la mañana, y hasta las doce y media, una, no... no paro, hago un montón de cosas. 
Moderadora- $\quad$ Te hacés el tiempo. Varias- Sí (Grupo 5).

Teresa (27 años, casada y madre de dos hijas pequeñas, empleada cesanteada de una fábrica) retoma esta idea tras la intervención de Margarita, y enfatiza la importancia de "hacerse el tiempo" personal por fuera de sus responsabilidades vinculadas con la reproducción doméstica: "En realidad uno se tiene que organizar, se tiene que armar el tiempo para uno mismo", señala. Su comentario dio lugar a un breve intercambio de opiniones acerca de un cambio generacional en las relaciones de género a partir de una mayor conciencia del derecho de las mujeres a disponer de tiempo propio para el cuidado de sí frente a las demandas domésticas y de cuidado de otros.:

Teresa: Aparte cuando uno está casado o tiene hijos, tiene que atender al marido, a los hijos, y siempre queda para lo último la mujer. iNo! En algún momento te tenés que hacer

Nidiaun

Eso

Tatianatiempo fue No, para antes, yo vos. ¿no? no.

Nidia- "Las de antes", las de antes que se dedicaban a sus hijos, a su casa, a su marido.

Isabel- Ahora también, pero es como que... Nidia- Ahora es como que más liberales somos las mujeres, algunas que otras (Grupo 5).

Articulan de este modo la idea de agencia temporal (Flaherty, 2011) y una visión crítica de relaciones de género más tradicionales. Siguiendo a Flaherty, este tipo de agencia implica la capacidad de organizar y distribuir el tiempo para uno y para los otros, y ejercer control sobre la experiencia temporal en la vida cotidiana: "estas prácticas de agencia tienden a ser intersticiales, porque uno busca tiempo para uno mismo en los pequeños espacios entre grandes bloques de tiempo que se asignan a las obligaciones (...) y resultan en formas terapéuticas de escapismo" (2011, p. 102-103). Por sus responsabilidades de cuidado, para algunas de las mujeres con hijos en edad escolar los bloques de tiempo personal se pueden liberar durante el período lectivo y se contraen durante las vacaciones. Por ejemplo, Victoria (casada, madre dos hijas pequeñas, empleada doméstica) concurría diariamente al gimnasio para hacer una rutina de levantamiento de pesas mientras sus hijas estaban en el colegio, "pero porque puedo hacerlo, porque tengo tiempo, cuando no tenga tiempo, bueno, no lo haré. Aprovecho que mis hijas están en el colegio y lo hago" (Grupo 6 ), mientras que Mara había suspendido sus caminatas matinales durante el verano para estar con sus hijos. Las responsabilidades de cuidado que las mujeres de clase popular no pueden delegar en otras a través de la compra de servicios de cuidado en el mercado (Esquivel, 2011; Batthyány, 2010) conducen a una práctica intermitente, pese a la voluntad y motivación personal para hacer una actividad corporal que valoran por sus beneficios psicofísicos.

Ejercer agencia temporal para el cuidado de sí también requiere disponer de dinero para uso personal. Gabriela (31 años, casada y madre de dos hijos pequeños) ejemplifica 
esta circunstancia. Ella había dejado de trabajar como empleada doméstica para cuidar a su hijo que tiene asma severa, pero había decidido retomar su actividad laboral una vez por semana para pagarse "el poli" y conservar el contacto con su empleadora. Hacía aquagym, pilates y gimnasia aeróbica cinco días a la semana. Al bienestar emocional y la posibilidad de tener un tiempo para ella, independientemente de sus tareas domésticas y de cuidado, se suman en su caso la posibilidad de socializar con otras personas ("conocer gente nueva, charlar") y el intento de bajar de peso:

Moderadora- ¿Y eso lo hacés porque te gusta?

Gabriela- Sí, por mí, sí en realidad por mí, porque antes que era el trabajo y el nene, el trabajo y el nene, [llevar al nene al] el hospital, viste que te agota. Entonces no me voy a trabajar más ahora, me voy a una vez a la semana, como para no perder la costumbre. (...) $Y$ ahora después me decidí, hace tres meses por ahí que me voy en el poli. Me voy todos los días y me despejo un poco.

hacés por vos.

Moderadora- Lo Porque en realidad no puedo bajar de peso (...) tengo que hacer actividad (...). Moderadora- Bueno. También se habla de la salud emocional, ¿no?, de la salud mental. ¿Eso es algo que ustedes también lo tienen en cuenta, le prestan atención? Vos un poco lo dijiste por lo de la... ¿no? De ir al poli, que te ayudaba a vos... Gabriela- Sí, sí, conocer gente nueva, charlar, porque hay veces que terminás, viste, y nos sentamos a charlar, a reírnos de lo que hicimos y eso, sí, te saca un poco de la vida cotidiana que uno tiene en casa, cuando uno tiene marido, hijos y eso, ayuda mucho salir un poco, porque si no estás todo el tiempo enfocada con los chicos, la casa, la comida, todo, entonces... A mí, por ejemplo, eso me da... No es que me aburre, pero como que... Moderadora- ¿Es como un espacio para vos? Gabriela- Claro (Grupo 3).

Otras participantes también enfatizan la importancia de compartir una actividad con amigos o familiares, no solo porque refuerza los vínculos interpersonales, sino también porque el apoyo social del entorno inmediato resulta un gran motivador para ponerse en movimiento. Por ejemplo, para Marta (55 años, casada, vive con tres hijos adultos y un nieto, voluntaria en un comedor comunitario) caminar durante los fines de semana hasta una localidad contigua significa, por sobre todo, un tiempo compartido con sus amigas:

Marta-Y el fin de semana a veces salimos caminando, con mis amigas. Nos vamos a la plaza de [una localidad cercana] a veces, y nos vamos caminando y venimos caminando. (...) $Y$, una hora nos lleva, porque nos vamos y venimos caminando, pero caminando rápido.

(...)

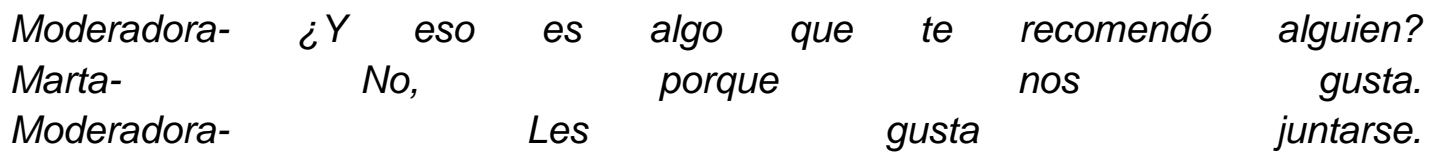
Marta- Sí, nos vamos porque a ellas les gusta ir caminando y yo me voy con ellas. Moderadora- $Y$ charlan, o sea es un momento para juntarse con amigas, eso sería. María- 


$$
\begin{aligned}
& \text { Moderadora- ¿Y también por algo de salud o...? } \\
& \text { María- No, no, por ir nomás (Grupo 1). }
\end{aligned}
$$

La posibilidad de disponer de tiempo contrasta con las limitaciones que enfrentaba Marta cuando era joven y vivía en la provincia de Corrientes; en esa etapa de su vida se veía impedida de realizar actividad física por el trabajo extradoméstico, el cuidado de sus nueve hijos y de la casa. El crecimiento de sus hijos y la migración a Buenos Aires significaron para ella poder salir a disfrutar de la caminata con sus amigas, "recién ahora estoy libre", comenta. El entorno de trabajo también puede propiciar hacer actividades conjuntas. En ese mismo grupo integrado por voluntarias de un comedor barrial, varias de las mujeres estaban planificando inscribirse juntas en un gimnasio, incentivadas por la coordinadora que lo hacía desde hacía un mes. Los relatos de las mujeres muestran cómo los círculos y contextos cotidianos de interacción y sociabilidad más próximos constituyen soportes colectivos para realizar actividad física de manera regular (Spence \& Lee, 2003).

\section{Estar en forma, prevenir enfermedades y mejorar condiciones de salud}

Por otro lado, al igual que Gabriela del Grupo 3, algunas participantes señalan el deseo de bajar de peso y motivaciones estéticas para realizar actividad física. Carolina (46 años, casada y madre de 4 hijos adolescentes) del Grupo 1 comenzó a ir al gimnasio luego de que dejó de caminar y ganó mucho peso. También es el caso de Lila (36 años), del Grupo 4, quien realiza aquagym y natación en el polideportivo "más que nada por estética y gusto". Un caso extremo es el de Juana (19 años) del Grupo 3, quien hace crossfit en un gimnasio privado, juega al hockey en un club social y corre en una plaza del barrio. Según la participante, ella llegó a tener una "adicción al deporte" junto con un cuadro cercano a la anorexia.

Prevenir una enfermedad, controlar factores de riesgo o mejorar una condición de salud también motiva a realizar ejercicio. Inés (55 años) del Grupo 4, que es asmática cuenta, "la natación me ayudó muchísimo me cambió la vida totalmente. Antes, cada dos por tres terminaba internada en el hospital con oxígeno, con Decadron”. En el mismo Grupo, Mirta (49 años) tiene un problema en la columna y cuando hace actividad física se siente muy aliviada. Sin embargo, con culpa, señala que normalmente no puede hacerlo por sus actividades laborales y domésticas. Marta (55 años) del Grupo 1 quisiera hacer gimnasia "por los huesos mismos, por sentirse bien". Por último, Margarita (52 años), la participante del Grupo 5 que realiza cuatro veces por semana actividades en el polideportivo para despejarse y olvidarse de sus problemas diarios, señala que la prevención es otro de los motivos. Con antecedentes familiares de diabetes, su médica le indicó realizar actividad física regularmente. Considera el hecho que ella no haya desarrollado la enfermedad -a diferencia de otros familiares de menor edad- como una evidencia de que el ejercicio ayuda a prevenir la diabetes.

Siguiendo a Lupton (1997), vemos que las motivaciones expresadas por las mujeres para realizar actividad física no solo se vinculan con los beneficios para la salud sino 
Revista Punto Género N. 15 Junio de 2021

ISSN 0719-0417 / 1- 24

también con el ideal estético del cuerpo femenino delgado.

\section{La actividad física cotidiana por el desplazamiento, la actividad laboral y el trabajo doméstico}

En algunos casos las mujeres elijen caminar o usar la bicicleta para desplazarse en el barrio, o bajarse antes del colectivo cuando vuelven de sus trabajos para hacer ejercicio, pero también lo hacen por sus necesidades diarias de traslado. Marta (55 años), del Grupo 1 , camina todos los días veinte cuadras para ir y volver del trabajo. En el caso de Mirta (49 años) del Grupo 4, durante un día promedio también camina para desplazarse por su trabajo como maestra estimuladora: "camino treinta minutos diarios pero todos repartidos. Camino bastante, pero por una cuestión de que todo me queda lejos (...) porque en todos los lugares de trabajo que tengo, que son diferentes tengo bastantes cuadras".

Para Blanca (24 años) del Grupo 5 ahorrarse el costo del transporte la lleva a ella y a su esposo a utilizar la bicicleta, "encima como está todo caro, así que cuando vamos a la iglesia vamos en bicicleta los dos, lo llevamos al nene atrás, y nos vamos pedaleando". A pesar de tratarse de un grupo de mujeres de sectores populares, el costo del transporte público no fue mencionado de manera recurrente como una razón para caminar o utilizar la bicicleta. Cabe señalar que los grupos fueron realizados en los años 2015 y 2016, y que con posterioridad se produjeron sucesivos recortes de los subsidios del Estado nacional al transporte público que generaron un fuerte aumento de su costo, por lo que es posible que en la actualidad el ahorro de dinero como razón para decidir caminar o utilizar la bicicleta sea más prominente.

Para muchas de las participantes, la actividad física también está vinculada con el desempeño laboral como trabajadoras manuales, y con su condición de amas de casa y madres de hijos pequeños. Florencia (47 años) empleada doméstica que participó del Grupo 6 señala que "en el trabajo subo y bajo [las escaleras]", mientras que para Margarita (52 años) del Grupo 5 que vende comida que cocina en su casa "es normal para mí, estar haciendo una actividad. Y esto, bueno, estoy todo el día en la cocina, y estoy todo el día haciendo verduras y cosas". Martina (24 años) del Grupo 6, al mirar el poster que recomendaba 30 minutos diarios de ejercicio, destaca la actividad física que realiza cotidianamente debido al cuidado de sus dos niños pequeños:

Martina-¿Ser mamá cuenta? Porque yo soy mamá. [Risas] ¿Ser mamá cuenta como ejercicio? (...) Y bueno, supongo que la maternidad también te tiene de acá para allá, ¿no? Por eso digo, me parece que... está bien, está un poco flojo todo, pero... [Risas, se refiere a su tonicidad muscular] Pero subo y bajo. (...) Yo me siento y me tengo que parar, me siento y me tengo que parar (Grupo 6). 


\section{Restricciones de género, temporales y económicas}

Una gran cantidad del tiempo de las participantes está dedicado al trabajo en el hogar y para el mercado, dejando poco tiempo libre para la actividad física recreativa. Dalma (19 años, vive con el novio, sin hijos) del Grupo 3 comenta que, a su trabajo como empleada doméstica, se suma la "ayuda" en el minimercado de sus suegros, resultando en una sobrecarga de trabajo diario que no le permite tener tiempo libre para ella. Además, Dalma prefiere no contradecir a su novio que no la "deja salir" a caminar, ni tampoco la acompaña, lo que contribuye a su aislamiento y muestra relaciones de género opresivas:

Moderadora- ¿Y con tu novio así salir a caminar?

Dalma- No, porque él en este momento está trabajando de lunes a lunes [en el minimercado]. (...). Yo más que nada por eso me quedo ahí, no tengo con quién salir tampoco. Sola no salgo.

Victoria- Tenés que salir sola, dejarlo a él que trabaje [risas].

Dalma- En realidad, digamos, no me deja (Grupo 3).

Clara (39 años, casada, madre de tres hijos adolescentes) del Grupo 4 también refiere a las limitaciones para liberar tiempo, por su trabajo como empleada doméstica dos veces por semana y la coordinación de un grupo de psicología social comunitaria que la hizo abandonar la asistencia al polideportivo del barrio, aunque lo compensa al ir caminando o en bicicleta al trabajo.

A veces las mujeres optan por no realizar actividad física para estar con sus seres queridos una vez concluida la jornada laboral y de capacitación, como es el caso de Susana (60 años, empleada doméstica, sin pareja) del Grupo 5, quien dejó de ir al polideportivo para compartir su escaso tiempo libre con sus hijos cuando ellos vuelven de sus trabajos y ella de un taller de costura al que asiste varias veces por semana:

Moderadora- $Y$ vos, Susana, decías que también vas al polideportivo.

Susana- No, iba, ahora no. Porque yo de acá salgo a las seis [del taller de capacitación], y ya voy a mi casa directamente a tomar mate, con mis hijos, mis dos hijos, que me están esperando, y de ahí ya si hay algo que comprar voy a comprar y ya viene la hora de cocinar y, viste, y ya... no (Grupo 5).

De manera similar, las actividades comunitarias también ocupan el tiempo personal que resta de las responsabilidades laborales y del hogar. Blanca (24 años, vive con el marido, su hijo de dos años y los suegros) comenta en el Grupo 5, "No, al poli no voy. Si tengo tiempo voy a los ensayos de la iglesia, al coro, y canto ahí, porque hago eso. Después estoy en mi casa, y bueno, a la mañana doy clases particulares, después ya tengo que cocinar, tiene que comer el nene, tiene que dormir". Mirta (49 años, vive con el esposo y sus cuatro hijos), que trabaja como maestra estimuladora en varios establecimientos, hace una referencia más general a cómo la falta de tiempo personal puede afectar el cuidado de la salud de las mujeres-madres:

Yo cuando hablaba de calidad de vida también pensaba en eso, la falta de tiempo 
para uno, ¿no? Especialmente pasa con las mamás, si tienen varios hijos, o si tienen muchos problemas vinculares o hacinamiento por ahí, y también las que trabajamos (...) pero también pasa que uno tiene poco tiempo para uno, para actividad física, para controles médicos, tenés poco tiempo, y todo eso influye también en la salud (Grupo 4).

El caso más extremo lo ejemplifica Patricia (25 años, vive con su marido, su hijo pequeño y su suegra) en quien se conjugan el agotamiento físico y la restricción de tiempo que le implica su trabajo como empleada doméstica, las demandas del cuidado del hogar y estar completando el bachillerato para adultos en el barrio, al que asiste por las tardes cuatro veces por semana hasta las 10 de la noche. No sólo no dispone de tiempo ni energía para utilizar el circuito aeróbico público en el barrio, sino que también sus prácticas de alimentación se ven muy restringidas por su ritmo de vida:

Patricia: Llego tres y cuarto [a mi casa]. Entre que acomodo las cosas ya se me fueron las ganas de comer, me bañé y ya tuve que venir para acá [al Bachillerato], o sea, ni tiempo a comer hice. $Y$ de noche ya... para mí no tiene sentido comer de noche, ya llego a las diez, diez y media, hasta que me pongo a acomodar, me baño, ya se hicieron las once, ya tengo que dormir porque al otro día a las seis de la mañana [me levanto]. No tengo tiempo $(\ldots)$

Moderadora- ¿Y ustedes también lo usan [al circuito aeróbico]? pone cara de ni loca]

[Patricia ¡Me salió del alma! No, yo no. No, no tengo tiempo. Moderadora- Pero los fines de semana está la placita, ¿no?, para ir. Patricia- Sí, no, no tengo tiempo, me dedico a la casa (Grupo 2).

Si bien aprovecha la caminata diaria desde y hacia el tren en CABA para ejercitar mientras se desplaza diariamente hacia su trabajo, ésta dista de ser recreativa ya que camina rápido por miedo a ser asaltada en el trayecto, lo que le produce estrés en lugar de disfrute y relajación:

Patricia: Son quince minutos caminando de ida, quince de vuelta, es una bajada, cuando voy es una subida, ya me ayuda. (...) Y a mí me da miedo andar sola, no me gusta, así que siempre ando así [hace señas de caminar rápido y mirando para todos lados], así que mejor, me ayuda el caminar rápido (Grupo 2).

Aunque realizadas de manera compulsiva, estas formas de estar en movimiento en algunos casos son vistas por ellas como una compensación de la falta de ejercicio vinculado con el ocio y el disfrute (Ainsworth, 2000).

Junto con la pobreza de tiempo y el agotamiento físico por la jornada laboral y en el hogar, el gasto económico que representa asistir a un gimnasio privado implica que para algunas mujeres esta opción sea inviable. "Es caro un gimnasio", afirma Laura (34 años) del Grupo 2, y destaca la necesidad de "hacer cuentas". En el mismo Grupo, Carla (41 años) coincide, "yo no lo podría hoy en día pagar". Como veremos, los espacios públicos para ejercitar y los subvencionados por el Estado disminuyen las barreras financieras para acceder a estas prácticas protectoras de la salud, siendo un recurso territorial clave para 
Revista Punto Género N. 15 Junio de 2021

ISSN 0719-0417 / 1- 24

las mujeres y sus familias.

\section{Falta de motivación personal}

Así como muchas mujeres resaltaron la importancia de "hacerse el tiempo" para su propio cuidado, en contraposición, la falta de voluntad surgió como una limitación. En el Grupo 1 ante la pregunta sobre qué opinaban del slogan "una vida saludable está a tu alcance" que proponía la gráfica que utilizamos como disparador en los grupos, Alicia (51 años) destacó:

Alicia- Y sí, creo que sí. Lo que pasa es que uno a veces no le pone la garra, la voluntad. Muchas veces uno se deja. Por ejemplo, a mí me gusta caminar, y a veces que voy capaz que a unas pocas cuadras y tomo un colectivo. Pero es por lo que queda más cómodo y todo eso, o no tener con quién ir, no querer ir sola también... (Grupo 1).

Aunque Alicia reconoce la importancia del apoyo social como incentivo, la frase "muchas veces uno se deja" indica que realizar actividad física forma parte de un ideal de autocuidado. En el Grupo 2, Laura (34 años, vive sola en una vivienda muy precaria) que padece asma, fuma y es obesa, dice no poder sostener las actividades que empieza por ser "fiaquieta", a lo que Mariana (21 años) le responde que su actitud denota una falta de amor por sí misma, "porque no te amás, porque si yo amo mi salud me voy a cuidar hasta lo último". Alcanzar un "cuerpo saludable" a través de regímenes de autocuidado y autocontrol se convierte en un proyecto vinculado a la construcción identitaria; siguiendo a Shilling "el cuerpo se transforma en un recurso y en un símbolo social" (1997, p. 5), El intercambio entre las participantes evidencia la internalización del mandato cultural del cuidado de sí como una responsabilidad individual, aun cuando las condiciones materiales de vida, la falta de soportes sociales y la propia condición de salud imponen fuertes restricciones para desarrollar prácticas de autocuidado.

\section{Recursos barriales y desventajas socio-territoriales}

Entre los recursos del barrio las participantes destacaron espacios públicos recientemente renovados, especialmente el circuito aeróbico y con aparatos que fue emplazado cerca del Centro de Salud, como una alternativa gratuita frente al gasto que implica un gimnasio o el polideportivo municipal:

Carla- a mí todos esos aparatos para hacer gimnasia que [se] hizo en el fondo a mí me ayudó para cuidar mi salud, porque un gimnasio no me lo puedo pagar. (...) Yo me voy todas las mañanas, ya es una terapia, camino hasta allá, que son veinte cuadras, hago gimnasia ahí y me vuelvo caminando otra vez hasta acá (Grupo 4).

El polideportivo constituye un recurso fundamental para las participantes, porque les permite acceder a ellas y a sus familias a una variada oferta de actividades a un costo que a varias de las participantes les resulta posible afrontar, comparándolo con el de los 
gimnasios privados. En el Grupo 5, Margarita (52 años) destaca la contención que el polideportivo brinda tanto a chicos como a grandes y la ventaja de que tenga una cuota accesible. No obstante, la poca disponibilidad de vacantes fue mencionada en los grupos como una barrera para la accesibilidad. El sostenimiento de la vacante resulta problemático, ya que debido a la alta demanda tres inasistencias consecutivas significan su pérdida. Por ejemplo, Carolina (46 años) del Grupo 1 perdió su vacante para natación, repitiéndose esta situación en dos años consecutivos:

Carolina- Natación es otra de las cosas que hacía. (...) Y cada vez que yo empezaba algo... dos años me pasó, empecé un año, y encima ahí no podés faltar, hasta tres faltas podés tener, después perdés la vacante. Y un año fui un par de meses, y después tenía una cosa, atrás otra cosa, atrás otra de [trabajo] y que no podía faltar, y tenía que faltar allá, entonces dejé. El siguiente año me pasó lo mismo y dejé de vuelta (Grupo 1).

La ubicación del polideportivo también genera problemas de accesibilidad para las mujeres que viven en las áreas más postergadas del barrio y no disponen de auto ni transporte público directo. Si lo hacen caminando, para llegar al polideportivo desde el lado "peor" del barrio tienen que cruzar, primero, el zanjón por alguna de las pasarelas o por el boulevard construido sobre la parte entubada del arroyo central, atravesar el lado "mejor" y luego cruzar la ruta por un puente peatonal con largas escaleras, "el único puente que hay [sobre la ruta] es el del polideportivo pero después que tenés que subir todas las escaleras y después bajar todas las escaleras" (Inés, Grupo 4). La carga de bebés y niños por tantas cuadras, así como también el uso de cochecitos para trasladarlos por algunas calles que se anegan cuando llueve y por donde también pasan automóviles y donde hay pequeños zanjones abiertos, conlleva un gran esfuerzo físico. Algunas calles aún son de tierra y tienen baches, y no cuentan con cordones ni veredas. A ello se suma la presencia constante de basura en las calles. Caminar en esas condiciones de abandono de calles y áreas que requieren de obras públicas para su mejora es una experiencia diaria de las mujeres pobres con hijos pequeños (Bostock, 2001). Constituyen barreras del ambiente físico a sortear que las afectan particularmente cuando dependen de caminar o del transporte público para sus desplazamientos cotidianos (Jirón Martínez, 2017). El mayor esfuerzo en el desplazamiento y el tiempo implicado, ya de por sí escaso para la mayoría de las participantes, puede resultar una experiencia estresante y desalentadora para hacer actividades en el polideportivo, "pero tenés que ir también. Yo camino, son dieciséis cuadras hasta el poli, voy caminando, vuelvo caminando, con el carrito [del hijo más chico], los llevo, los traigo, lo tenés que hacer" (Inés, Grupo 4).

Se suman a las desventajas territoriales físicas, la inseguridad que lleva a las mujeres a evitar estar en la calle en horarios que consideran peligrosos. El riesgo a ser víctimas de violencia interpersonal y robos es un factor limitante para la realización de actividad física en el espacio público. En el Grupo 3, cuyas participantes vivían en una de las áreas más vulnerables, las mujeres comentaron que el barrio les resulta inseguro a partir del atardecer. Juana (19 años), realiza actividad física en una plaza cercana sólo si es bajo la vigilancia de su padre: "mi papá me espera en el auto y yo corro unas vueltas y me voy 
con ér'. La experiencia de Dalma (19 años) también muestra que no tener compañía masculina es un impedimento para caminar por lugares donde pueden ser víctimas de molestias y agresiones:

Moderadora- ¿Y vos, Dalma, decías, cuando preguntaba si podían caminar tranquilas, dijiste "no, no", ¿no?

Dalma- No, porque yo, la verdad, si salgo, salgo sola, y no, prefiero quedarme en casa por la seguridad misma. Porque salir a esa hora como ella dice también... Moderadora- ¿Qué sería, a partir de qué hora más o menos, es inseguro? Dalma- $Y$, cuando empieza a anochecer, siete o algo. (...) Porque a veces se juntan en esquinas, viste, $y$ no te dejan pasar. Moderadora- $Y$ son los propios chicos que viven acá, o la gente...

Dalma- Sí, claro. Se juntan ahí, se drogan, y no te dan ganas de pasar también (Grupo 3).

Sólo una participante aseguró que podía salir sola a caminar por la plaza del barrio de noche sin problemas: "caminaba una hora y me venía (...) por ahí más a la nochecita también, no hay problema” (Carolina, 46 años, Grupo 1). La restricción temporal para circular y hacer uso de espacios públicos para la caminata y el ejercicio puede redundar en un empobrecimiento personal y social, siendo, como lo indican los relatos, un impedimento que afecta especialmente a las mujeres más jóvenes (Segovia y Rico, 2017).

\section{Discusión y conclusiones}

Las participantes de los grupos coinciden en la valoración de la actividad física recreativa como una práctica que es "buena para ellas" desde una concepción integral de la salud que abarca aspectos físicos, mentales y emocionales. La actividad física recreativa constituye una oportunidad para tomar distancia de sus responsabilidades cotidianas y tiene el efecto de "paréntesis" de sus preocupaciones y ocupaciones diarias. La posibilidad de pasar tiempo libre con amigas o conocer gente nueva a través de actividades colectivas también es un beneficio que les brinda hacer actividad física. Efectivamente, socializar y entablar nuevas relaciones interpersonales es una dimensión de la salud y del desarrollo personal cuando se la considera en su integralidad (Burnett, 2002; World Health Organization, 2018).

La pobreza de tiempo restringe sus posibilidades de hacer ejercicio recreativo de manera regular, especialmente para las mujeres que viven en pareja y son madres de niños pequeños, por el tiempo que les insume el trabajo doméstico y el cuidado familiar -además del trabajo para el mercado. Sin embargo, se destaca en los relatos de las participantes la búsqueda de ejercer agencia temporal (Flaherty, 2011) para liberar tiempo para el cuidado de sí, lo que, al parecer de algunas de ellas, indica un cambio generacional en las relaciones de género hacia una mayor autonomía y menor subordinación en las relaciones de pareja. La búsqueda de ejercer agencia temporal para hacer una actividad que les brinda bienestar personal resulta en un empoderamiento frente a las relaciones de género subordinantes 
que imponen desiguales cantidades de tiempo libre para mujeres y varones, y relegan las necesidades de cuidado de sí las mujeres con hijos pequeños (Burnett, 2002). La valoración del tiempo personal para el autocuidado que expresaron las mujeres que participaron de nuestro estudio contradice la idea de "lujo" que remarcan Tortolero et al. (1999), en relación a la posibilidad de hacer actividad física recreativa para las mujeres que viven en condiciones de fuertes restricciones materiales y con una alta carga de responsabilidades domésticas y de cuidado. Aunque la referencia fue marginal en las discusiones grupales, relaciones opresivas de género son también parte de la realidad de algunas mujeres jóvenes que dependen del "permiso" de sus parejas para realizar caminatas en el escaso tiempo libre del que disponen, o bien de la compañía masculina para apropiarse del espacio público y sentirse más seguras.

Las referencias a la falta de motivación y voluntad de algunas participantes para hacer ejercicio ponen de manifiesto un marco normativo desde el cual se evalúan a sí mismas y sus cuerpos, que es a veces acompañado por un sentimiento de culpa, y que conforma un entramado moral entre la realización de actividad física orientado por el esfuerzo, el cuidado de la salud y la valoración de sí mismas (McDermott, 2011; Lupton, 1997). Nuestro estudio muestra que el imperativo cultural y normativo no solo informa las motivaciones para hacer actividad física de mujeres de clase media (Ballesteros et al, 2016; Freidin, 2017a) sino también de las de clase popular. Vemos cómo la responsabilidad individual por la salud puede tener como contrapunto la culpabilización de la víctima (Lupton, 1997; Crawford, 2006) para mujeres cuyas condiciones de vida son muy desventajosas.

Más allá de la actividad física recreativa, las participantes realizan ejercicio por su actividad ocupacional, el trabajo en el hogar, el cuidado de los niños y las necesidades de transporte. En general, no tienen automóvil propio y viven en la parte con menor conectividad del barrio. Caminar por calles y áreas que requieren de obras públicas para su mejora es una experiencia diaria para las mujeres con hijos pequeños que viven en las áreas más postergadas del barrio, en consonancia con el estudio de Bostock, , en el que concluye que "para algunos segmentos de la población caminar es compulsivo y una fuente de fatiga física y estrés psicosocial (...). No sólo los bajos ingresos obligan a las mujeres a caminar, se ven forzadas a hacerlo a través de áreas con basura y abandonadas" (2001, p. 11-12). Se trata de barreras del ambiente físico a sortear que afectan particularmente a las mujeres de menores recursos que dependen de caminar o del transporte público para sus desplazamientos cotidianos (Jirón Martínez, 2017). Asimismo, aunque caminen por su barrio y otros por su actividad laboral, algunas de las participantes de los grupos sienten miedo de sufrir ataques callejeros y por eso lo hacen aceleradamente. Así, por sus condiciones de vida, de trabajo y las actividades propias de la vida cotidiana, las mujeres realizan ejercicio físico que no necesariamente está asociado con beneficios para la salud, sino con el agotamiento físico y emocional, como lo señala Bostock (2001). Aunque de carácter compulsivo, estas formas de estar en movimiento, en algunos casos, son vistas por ellas como una compensación ante la falta de ejercicio recreativo, en coincidencia con el estudio de Ainsworth (2000). 
Los espacios públicos para ejercitar al aire libre y los subvencionados por el Estado constituyen recursos territoriales claves para las mujeres y sus familias. Las experiencias de las participantes de los grupos ponen de relieve la importancia de incrementar la inversión pública en infraestructura y equipamiento para disminuir las barreras económicas y territoriales para la práctica de ejercicio físico y deporte (Moore et al, 2008; Ståhl et al., 2001; World Health Organization, 2018), y ejemplifican modalidades de uso del espacio público en los sectores populares identificados en estudios cuantitativos con encuestas nacionales (Ballesteros \& Freidin, 2019). Sin embargo, pese a la disponibilidad de infraestructura, el aprovechamiento de recursos comunitarios como el polideportivo se ve restringido en ocasiones por las responsabilidades domésticas y laborales que les dificulta mantener la vacante. Destacamos frente a esta limitante, que afecta principalmente a las mujeres de clase popular con niños pequeños, la importancia de implementar políticas públicas con una perspectiva de género y programas específicos para promover la actividad física incorporando la dimensión del cuidado infantil (Ballesteros \& Freidin, 2019; Ballesteros et al., 2021).

El uso del espacio público para realizar ejercicio recreativo también se ve limitado por el riesgo de ser asaltadas o acosadas sexualmente, especialmente para las mujeres más jóvenes. La experiencia de inseguridad restringe la circulación por el barrio a determinadas horas, o a un menor radio, lo cual puede repercutir en su bienestar y en la transformación de zonas segregadas en zonas de alta vulnerabilidad determinadas por el género (Jirón Martínez, 2017; Segovia \& Rico, 2017).

\section{Bibliografía}

Abichahine, H. \& Veenstra, G. (2017): "Inter-categorical intersectionality and leisure-based physical activity in Canada", en Health Promotion International, Vol. 32, No. 4, p. 691-701.

Ainsworth, B (2000): "Issues in the assessment of physical activity in women", en Research quarterly for exercise and sport, Vol. 71, No. 2, p. 37-42.

Allen-Collinson, J. (2011): "Running Embodiment, Power and Vulnerability: Notes toward a Feminist Phenomenology of Female Running", en: Kennedy y Markula (comp.): Women and exercise: the body, health and consumerism, p. 280-298. New York: Routledge.

Ballesteros, M., Freidin, B., Krause, M. \& Borda, P. (2016): "La práctica de actividad física entre varones y mujeres de clase media en el AMBA: motivaciones, percepción de beneficios y limitaciones para su continuidad", en Educación Física y Ciencia, Vol. 18, No. 2 [online]. Disponible en http://www.efyc.fahce.unlp.edu.ar/article/view/EFyCa009/ 
Ballesteros, M. \& Freidin, B. (2019): "Desigualdades sociales en la realización de distintas modalidades de actividad física en Argentina”, en Educación Física y Ciencia, Vol. 21, No. 1 [online]. Disponible en https://www.efyc.fahce.unlp.edu.ar/article/view/EFyCe067/10677

Ballesteros, M., Betina F. \& Agustín D. (2021): "Determinantes sociales asociados a la actividad física recreativa y para el cuidado de la salud. República Argentina 2009y 2018". Revista Lecturas de Educación Física y Deporte, Vol. 26, No. 276, 2-19

Batthyány, K. (2010): Género, cuidados familiares y uso del tiempo. Informe final de investigación. Montevideo: UNIFEM.

Boersma, M. (2016): "Low incomes, fast lives? Exploring everyday temporalities of Filipina domestic workers in Hong Kong", en Time \& Society, Vol. 25, No. 1, p. 117-137.

Bostock, L. (2001): "Pathways of disadvantage? Walking as a mode of transport among low income mothers", en Health \& Social Care in the Community, Vol. 9, No. 1, p. 11-18.

Brady, B. \& Nies, M. (1999): "Health-promoting lifestyles and exercise: A comparison of older African American women above and below poverty level", en Journal of Holistic Nursing, Vol. 17, No. 2, p. 197-207.

Burnett, C. (2002): "Women, poverty and sport: A South African scenario", en Women in Sport and Physical Activity Journal, Vol. 11, No. 1, p. 23-47.

Carey, M. \& Asbury, J. (2012) : Focus Group Research. Walnut Creek: Lef Cost Press.

Crawford, R. (2006): "Health as a meaningful social practice", Health, Vol.10, No.4, p. 401420.

Conrad, P. (1994): "Wellness as Virtue: Morality and the Pursuit of Health", Culture, Medicine and Psychiatry, Vol.18, p. 385-401.

Cockerham, W. (2010): "Health Lifestyles: Bringing Structure Back". En Cockerham (Ed), The New Blackwell Companion to Medical Sociology. London: Wiley-Blackwell.

Diez Roux, A. \& Mair, C. (2010): "Neighborhoods and Health", en Annals of the New York Academy of Sciences", Vol. 1186, No. 1, p. 125-145 [online].Disponible en https://nyaspubs.onlinelibrary.wiley.com/doi/epdf/10.1111/j.1749-

6632.2009.05333.x 
Esquivel, V. (2011): La economía del cuidado en América latina: Poniendo a los cuidados en el centro de la agenda. PNUD.

Flaherty, M. (2011): The textures of Time: Agency and Temporal experience. Filadelfia: Temple University Press.

Freidin, B. (2017a) : "Introducción”, en Freidin (Coordinadora) Cuidar la salud: mandatos culturales y prácticas cotidianas de la clase media en Buenos Aires. Buenos Aires: Editorial Imago Mundi.

Freidin, B. (2017b): "El uso de despliegues visuales en el análisis de datos cualitativos: ¿Para qué y cómo los diseñamos?”. En Borda, Dabenigno, Freidin, Guelman, Cuaderno de Metodología No.2 ¿Cómo se hace?: Estrategias de análisis cualitativo. Buenos Aires: Instituto Gino Germani.

Freidin, B. (2016): "Revisando el uso de los grupos focalizados en la investigación social", Revista Latinoamericana de Metodología, Vol. 6, No 1, p.1-30.

Freidin, B., Wilner, A., Ballesteros, M. y Krause, M. (2018): Salud, riesgo ambiental y territorio: las experiencias de mujeres que viven en un barrio de clase popular en la periferia norte de Buenos Aires. Buenos Aires: Teseo.

García F., Manuel Y Llopis Goig, R. (2017): "Estructura social de la práctica deportiva”, en García Ferrando, Puig Barata, Lagardera Otero, LlopisGoig, Vilanova Soler (comp.): Sociología del deporte. Madrid: Alianza Editorial.

Gómez Rojas, G. \& Riveiro, M. (2015): “El género en la relación entre clase social y estilo de vida: una mirada a través del tiempo libre", en Millcayac-Revista Digital de Ciencias Sociales, Vol. 2, No. 2, p. 79-106 [online]. Disponible en http://revistas.uncu.edu.ar/ojs/index.php/millcadigital/article/view/300/150

Hargreaves, J. (1993): "Problemas y promesas en el ocio y los deportes femeninos", en Brohm, Bourdieu, Dunning, Hargreaves, Todd, Young (comp.): Materiales de sociología del deporte. Madrid: Ediciones La Piqueta.

Hormiga-Sánchez, C. (2015): "Perspectiva de género en el estudio de la práctica de actividad física”, en Revista Ciencias de la Salud, Vol. 13, № 2, p. 233-248, [On Line]. Disponible en: dx.doi.org/10.12804/revsalud13.02.2015.08

Huberman, M \& Miles, M. (1994): "Data management and analysis methods", en Denzin, Lincoln (eds.), Handbook of Qualitative Research, p. 428-444. London: Sage publications. 
lyer, A.; Sen, G. \& Östlin, P. (2008): "The intersections of gender and class in health status and health care", en Global public health, Vol. 3, № 1, p. 13-24.

Jirón Martínez, P. (2017): "Planificación urbana y del transporte a partir de relaciones de interdependencia y movilidad del cuidado", en Rico, María N. y Segovia, Olga (comp.): ¿Quién cuida en la ciudad? Aportes para políticas urbanas de igualdad, p. 405-432. Santiago: CEPAL. Disponible en: https://www.cepal.org/es/publicaciones/42424-quien-cuida-la-ciudad-aportespoliticas-urbanas-igualdad

Lehner, M. \& Ponce, M. (2018): “El auto cuidado de la salud”, en Findling, Liliana y López, Elsa (comp.): Cuidados y familias: los senderos de la solidaridad intergeneracional, p 113-132. Buenos Aires: Teseo.

Linetzky, B.; De Maio, F.; Ferrante, D.; Konfino, J. \& Boissonnet, C. (2013): "Sex-stratified socio-economic gradients in physical inactivity, obesity, and diabetes: evidence of short-term changes in Argentina", en International journal of public health, Vol. 58, № 2, p. 277-284.

Lupton, D. (1997): The Imperative of Health: Public Health and the Regulated Body, London: Sage.

Markula, P. y Kennedy, E. (2011): "Beyond binaries: contemporary approaches to women and exercise", en Kennedy, Eileen y Markula, Pirkko (comp.): Women and exercise: the body, health and consumerism, p 1-26. New York: Routledge.

Martín, M.; Soler, S. y Vilanova, A. (2017): “Género y deporte”, en García Ferrando, Manuel; Puig Barata, Núria; Lagardera Otero, Francisco; Llopis Goig, Ramón y Vilanova Soler, Ana (comp.) Sociología del deporte, p. 97-123. Madrid: Alianza.

McDermott, L. (2011): ““Doing Something that's Good for Me”: Exploring Intersections of Physical Activity and Health", Kennedy, Eileen y Markula, Pirkko (comp.): Women and exercise: the body, health and consumerism, p. 197-226. New York: Routledge.

Moore, L., Diez R., A., Evenson, K..; McGinn, A. \& Brines, S. (2008): "Availability of recreational resources in minority and low socioeconomic status areas", en American Journal of Preventive Medicine, Vol. 34, № 1, p. 16-22, [On Line]. Disponible en: http://citeseerx.ist.psu.edu/viewdoc/download?doi=10.1.1.660.6855\&rep=rep1 \&type $=$ pdf 
Mosquera González, M. (2014): ¿LLas mujeres no hacen deporte porque no quieren? ¿Los hombres practican el deporte que quieren? El género como variable de análisis de la práctica deportiva de las mujeres y de los hombres", en IV Ciclo de conferencias Xénero, Actividade Física e Deporte (2012-2013), p. 71-84, [On Line]. Disponible en: http://ruc.udc.es/dspace/handle/2183/13594

ODSA (2017): Desarrollo humano e integración social en la argentina urbana 2010-2016. Documento estadístico. Disponible en: https://repositorio.uca.edu.ar/bitstream/123456789/8197/1/desarrollo-humanointegracion-social-estadistico.pdf

Segovia, O. \& Rico, M. (2017): “¿Cómo vivimos la ciudad? Hacia un nuevo paradigma urbano para la igualdad de género", en Rico, María N. y Segovia, Olga (comp.): ¿Quién cuida en la ciudad? Aportes para políticas urbanas de igualdad, p. 4170. Santiago: CEPAL. Disponible en: https://www.cepal.org/es/publicaciones/42424-quien-cuida-la-ciudad-aportespoliticas-urbanas-igualdad

Shilling, C. (1997): The Body and Social Theory; London, Sage

Spence, J. \& Rebecca L. (2003): "Toward a comprehensive model of physical activity". Psychology of Sport and Exercise 4(1): 7-24.

Ståhl, T., Rütten, A., Nutbeam, D., Bauman, A., Kannas, L.,; Abel, T., Lüschen, G., Rodriquez, D., Vinck, J. \& Van der Zee, J. (2001): "The importance of the social environment for physically active lifestyle-results from an international study", en Social science \& medicine, Vol. 52, № 1, p. 1-10, [On Line]. Disponible en: https://doi.org/10.1016/S0277-9536(00)00116-7

Strazdins, L.; Welsh, J.; Korda, R.; Broom, D. \& Paolucci, F. (2015): "Not all hours are equal: could time be a social determinant of health?", en Sociology of Health and IIIness, Vol. 38, № 1, p. 21-42, [On Line]. Disponible en: https://doi.org/10.1111/1467-9566.12300

Tortolero, S., Mâsse, L., Fulton, J., Torres, I. \& Kohl, H.. (1999): "Assessing physical activity among minority women: Focus group results”, en Women's Health Issues, Vol. 9, № 3, p. 135-142.

World Health Organization (2018): Global action plan on physical activity 2018-2030: more active people for a healthier world. Disponible en: 
Revista Punto Género N. 15 Junio de 2021

ISSN 0719-0417 / 1- 24

https://apps.who.int/iris/bitstream/handle/10665/272722/9789241514187eng.pdf 